\title{
Quantum games and quantum strategies
}

\author{
Jens Eisert, ${ }^{1}$ Martin Wilkens, ${ }^{1}$ and Maciej Lewenstein ${ }^{2}$ \\ ${ }^{1}$ Institut für Physik, Universität Potsdam, 14469 Potsdam, Germany \\ ${ }^{2}$ Institut für Theoretische Physik, Universität Hannover, 30167 Hannover, Germany
}

(Dated: March 16, 2020)

\begin{abstract}
We investigate the quantization of non-zero sum games. For the particular case of the Prisoners' Dilemma we show that this game ceases to pose a dilemma if quantum strategies are allowed for. We also construct a particular quantum strategy which always gives reward if played against any classical strategy.
\end{abstract}

One might wonder what games and physics could have possibly in common. After all, games like chess or poker seem to heavily rely on bluffing, guessing and other activities of unphysical character. Yet, as was shown by von Neumann and Morgenstern [1], conscious choice is not essential for a theory of games. At the most abstract level, game theory is about numbers that entities are efficiently acting to maximize or minimize [2]. For a quantum physicist it is then legitimate to ask what happens if linear superpositions of these actions are allowed for, that is if games are generalized into the quantum domain.

There are several reasons why quantizing games may be interesting. First, classical game theory is a well established discipline of applied mathematics [2] which has found numerous applications in economy, psychology, ecology and biology [2, 3]. Since it is based on probability to a large extend, there is a fundamental interest in generalizing this theory to the domain of quantum probabilities. Second, if the "Selfish Genes" [3] are reality, we may speculate that games of survival are being played already on the molecular level where quantum mechanics dictates the rules. Third, there is an intimate connection between the theory of games and the theory of quantum communication. Indeed, whenever a player passes his decision to the other player or the game's arbiter, he in fact communicates information, which - as we live in a quantum world - is legitimate to think of as quantum information. On the other hand it has recently been transpired that eavesdropping in quantum-channel communication [4-6] and optimal cloning [7] can readily be conceived a strategic game between two or more players, the objective being to obtain as much information as possible in a given set-up. Finally, quantum mechanics may well be useful to win some specially designed zero-sum unfair games, like PQ penny flip, as was recently demonstrated by Meyer [8], and it may assure fairness in remote gambling [9].

In this letter we consider non-zero sum games where - in contrast to zero-sum games - the two players no longer appear in strict opposition to each other, but may rather benefit from mutual cooperation. A particular instance of this class of games, which has found widespread applications in many areas of science, is the Prisoners' Dilemma. In the Prisoners' Dilemma, each of the two players, Alice and Bob, must independently decide whether she or he chooses to defect (strategy $D$ ) or cooperate (strategy $C$ ). Depending on their decision taken, each player receives a certain pay-off - see Tab. . The objective of each player is to maximize his or her individual pay-off. The catch of the dilemma is that $D$ is the dominant

\begin{tabular}{|l|cc|}
\hline & Bob: $C$ & Bob: $D$ \\
\hline Alice: $C$ & $(3,3)$ & $(0,5)$ \\
Alice: $D$ & $(5,0)$ & $(1,1)$ \\
\hline
\end{tabular}

TABLE I. Pay-off matrix for the Prisoners' Dilemma. The first entry in the parenthesis denotes the pay-off of Alice and the second number is Bob's pay-off. The numerical values are chosen as in [3]. Referring to Eq. (2) this choice corresponds to $r=3$ ("reward"), $p=1$ ("punishment"), $t=5$ ("temptation"), and $s=0$ ("sucker's payoff").

strategy, that is, rational reasoning forces each player to defect, and thereby doing substantially worse than if they would both decide to cooperate [10]. In terms of game theory, mutual defection is also a Nash equilibrium [2]: in contemplating on the move $D D$ in retrospect, each of the players comes to the conclusion that he or she could not have done better by unilaterally changing his or her own strategy [11].

In this paper we give a physical model of the Prisoners' Dilemma, and we show that - in the context of this model the players escape the dilemma if they both resort to quantum strategies. Moreover, we shall demonstrate that (i) there exists a particular pair of quantum strategies which always gives reward and is a Nash equilibrium and (ii) there exist a particular quantum strategy which always gives at least reward if played against any classical strategy.

The physical model consists of (i) a source of two bits, one bit for each player, (ii) a set of physical instruments which enable the player to manipulate his or her own bit in a strategic manner, and (iii) a physical measurement device which determines the players' pay-off from the state of the two bits. All three ingredients, the source, the players' physical instruments, and the pay-off physical measurement device are assumed to be perfectly known to both players.

The quantum formulation proceeds by assigning the possible outcomes of the classical strategies $D$ and $C$ two basis vectors $|D\rangle$ and $|C\rangle$ in the Hilbert space of a two-state system, i.e., a qubit. At each instance, the state of the game is described by a vector in the tensor product space which is spanned by the classical game basis $|C C\rangle,|C D\rangle,|D C\rangle$ and $|D D\rangle$, where the first and second entry refer to Alice's and Bob's qubit, respectively.

The board of our quantum-game is depicted in Fig. 1, it can in fact be considered a simple quantum network [12] with sources, reversible one-bit and two-bit gates and sinks. Note that the complexity is minimal in this implementation as the players' decisions are encoded in dichotomic variables. 


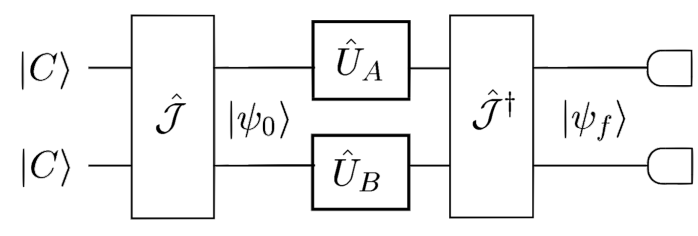

FIG. 1. The setup of a two player quantum game.

We denote the game's initial state vector by $\left|\psi_{0}\right\rangle=\hat{\mathcal{J}}|C C\rangle$, where $\hat{\mathcal{J}}$ is a unitary operator which is known to both players. For fair games $\hat{\mathcal{J}}$ must be symmetric with respect to the interchange of the two players. The strategies are executed on the distributed pair of qubits in the state vector $\left|\psi_{0}\right\rangle$. Strategic moves of Alice and Bob are associated with unitary operators $\hat{U}_{A}$ and $\hat{U}_{B}$, respectively, which are chosen from a strategic space $S$. The independence of the players dictates that $\hat{U}_{A}$ and $\hat{U}_{B}$ operate exclusively on the qubits in Alice's and Bob's possession, respectively. The strategic space $S$ may therefore be identified with some subset of the group of unitary $2 \times 2$ matrices.

Having executed their moves, which leaves the game in a state vector $\left(\hat{U}_{A} \otimes \hat{U}_{B}\right) \hat{\mathcal{J}}|C C\rangle$, Alice and Bob forward their qubits for the final measurement which determines their payoff. The measurement device consists of a reversible two-bit gate $\tilde{\mathcal{J}}$ which is followed by a pair of Stern Gerlach type detectors. The two channels of each detector are labeled by $\sigma=C, D$. With the proviso of subsequent justification we set $\tilde{\mathcal{J}}=\hat{\mathcal{J}}^{\dagger}$, such that the final state vector $\left|\psi_{f}\right\rangle=\left|\psi_{f}\left(\hat{U}_{A}, \hat{U}_{B}\right)\right\rangle$ of the game prior to detection is given by

$$
\left|\psi_{f}\right\rangle=\hat{\mathcal{J}}^{\dagger}\left(\hat{U}_{A} \otimes \hat{U}_{B}\right) \hat{\mathcal{J}}|C C\rangle .
$$

The subsequent detection yields a particular result, $\sigma \sigma^{\prime}=C D$ say, and the pay-off is returned according to the corresponding entry of the pay-off matrix. Yet quantum mechanics being a fundamentally probabilistic theory, the only strategic notion of a pay-off is the expected pay-off. Alice's expected pay-off is given by

$$
\$_{A}=r P_{C C}+p P_{D D}+t P_{D C}+s P_{C D},
$$

where $P_{\sigma \sigma^{\prime}}=\left|\left\langle\sigma \sigma^{\prime} \mid \psi_{f}\right\rangle\right|^{2}$ is the joint probability that the channels $\sigma$ and $\sigma^{\prime}$ of the Stern-Gerlach type devices will click. Bob's expected pay-off is obtained by interchanging $t \leftrightarrow s$ in the last two entries (for numerical values of $r, p, t, s$ see Tab. ). Note that Alice's expected pay-off $\$_{A}$ not only depends on her choice of strategy $\hat{U}_{A}$, but also on Bob's choice $\hat{U}_{B}$.

It proves to be sufficient to restrict the strategic space to the 2-parameter set of unitary $2 \times 2$ matrices

$$
\hat{U}(\theta, \phi)=\left(\begin{array}{cc}
e^{i \phi} \cos \theta / 2 & \sin \theta / 2 \\
-\sin \theta / 2 & e^{-i \phi} \cos \theta / 2
\end{array}\right)
$$

with $0 \leq \theta \leq \pi$ and $0 \leq \phi \leq \pi / 2$. To be specific, we associate the strategy "cooperate" with the operator

$$
\hat{C}:=\hat{U}(0,0), \quad \hat{C}=\left(\begin{array}{ll}
1 & 0 \\
0 & 1
\end{array}\right),
$$

while the strategy "defect" is associated with a spin-flip,

$$
\hat{D}:=\hat{U}(\pi, 0), \quad \hat{D}=\left(\begin{array}{cc}
0 & 1 \\
-1 & 0
\end{array}\right) .
$$

In order to guarantee that the ordinary Prisoners' Dilemma is faithfully represented, we impose the subsidiary conditions

$$
[\hat{\mathcal{J}}, \hat{D} \otimes \hat{D}]=0,[\hat{\mathcal{J}}, \hat{D} \otimes \hat{C}]=0,[\hat{\mathcal{J}}, \hat{C} \otimes \hat{D}]=0 .
$$

These conditions together with the identification $\tilde{\mathcal{J}}=\hat{\mathcal{J}}^{\dagger}$ imply that for any pair of strategies taken from the subset $S_{0}:=\{\hat{U}(\theta, 0) \mid \theta \in[0, \pi]\}$, the joint probabilities $P_{\sigma \sigma^{\prime}}$ factorize, $P_{\sigma \sigma^{\prime}}=p_{A}^{(\sigma)} p_{B}^{\left(\sigma^{\prime}\right)}$, where $p^{(C)}=\cos ^{2}(\theta / 2)$ and $p^{(D)}=1-p^{(C)}$. Identifying $p^{(C)}$ with the individual preference to cooperate, we observe that condition (6) in fact assures that the quantum Prisoners' Dilemma entails a faithful representation of the most general classical Prisoners' Dilemma, where each player uses a biased coin in order to decide whether he or she chooses to cooperate or to defect [13]. Of course, the entire set of quantum strategies is much bigger than $S_{0}$, and it is the quantum sector $S \backslash S_{0}$ which offers additional degrees of freedom that can be exploited for strategic purposes. Note that our quantization scheme applies to any two player binary choice symmetric game and - due to the classical correspondence principle Eq. (6) - is to a great extent canonical.

Factoring out Abelian subgroups which yield nothing but a reparametrization of the quantum sector of the strategic space $S$, a solution of Eq. (6) is given by

$$
\hat{\mathcal{J}}=\exp (-i \gamma \hat{D} \otimes \hat{D} / 2),
$$

where $\gamma \in[0, \pi / 2]$ is a real parameter. In fact, $\gamma$ is a measure for the game's entanglement. For a separable game $\gamma=0$, and the joint probabilities $P_{\sigma \sigma^{\prime}}$ factorize for all possible pairs of strategies $\hat{U}_{A}, \hat{U}_{B}$. Fig. 2 shows Alice's expected pay-off for $\gamma=0$. As can be seen in this figure, for any of Bob's choices $\hat{U}_{B}$ Alice's pay-off is maximized if she chooses to play $\hat{D}$. The game being symmetric, the same holds for Bob and $\hat{D} \otimes \hat{D}$ is the equilibrium in dominant strategies. Indeed, separable games do not display any features which go beyond the classical game.

The situation is entirely different for a maximally entangled game $\gamma=\pi / 2$. Here, pairs of strategies exist which have no counterpart in the classical domain, yet by virtue of Eq. (6) the game behaves completely classical if both players decide to play $\phi=0$. For example, $P_{C C}=$ $\left|\cos \left(\phi_{A}+\phi_{B}\right) \cos \left(\theta_{A} / 2\right) \cos \left(\theta_{B} / 2\right)\right|^{2}$ factorizes on $S_{0} \otimes S_{0}$ (i.e., $\phi_{A}=\phi_{B}=0$ fixed), but exhibits non-local correlations otherwise. In Fig. 3 we depict Alice's pay-off in the Prisoners' Dilemma as a function of the strategies $\hat{U}_{A}, \hat{U}_{B}$. Assuming Bob chooses $\hat{D}$, Alice's best reply would be

$$
\hat{Q}:=\hat{U}(0, \pi / 2), \quad \hat{Q}=\left(\begin{array}{cc}
i & 0 \\
0 & -i
\end{array}\right),
$$

while assuming Bob plays $\hat{C}$ Alice's best strategy would be defection $\hat{D}$. Thus, there is no dominant strategy left for Alice. 


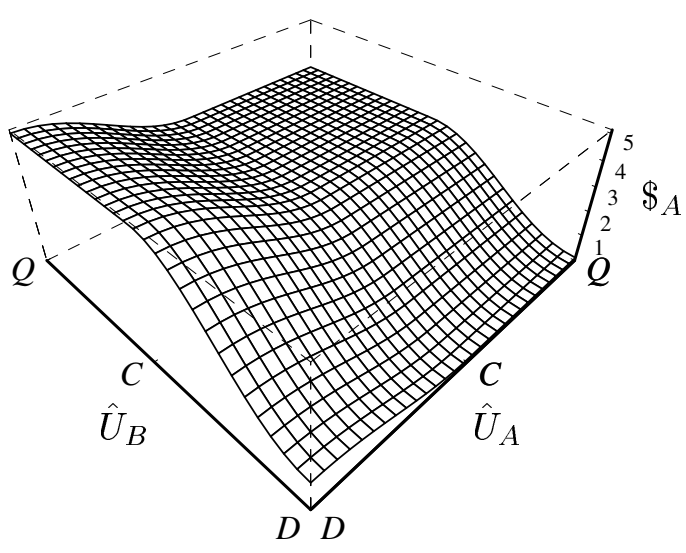

FIG. 2. Alice's pay-off in a separable game. In this and the following plot we have chosen a certain parametrization such that the strategies $\hat{U}_{A}$ and $\hat{U}_{B}$ each depend on a single parameter $t \in[-1,1]$ only: we set $\hat{U}_{A}=\hat{U}(t \pi, 0)$ for $t \in[0,1]$ and $\hat{U}_{A}=\hat{U}(0,-t \pi / 2)$ for $t \in[-1,0)$ (same for Bob). Defection $\hat{D}$ corresponds to the value $t=1$, cooperation $\hat{C}$ to $t=0$, and $\hat{Q}$ is represented by $t=-1$.

The game being symmetric, the same holds for Bob, i.e., $\hat{D} \otimes \hat{D}$ is no longer an equilibrium in dominant strategies.

Surprisingly, $\hat{D} \otimes \hat{D}$ even ceases to be a Nash equilibrium as both players can improve by unilaterally deviating from the strategy $\hat{D}$. However, concomitant with the disappearance of the equilibrium $\hat{D} \otimes \hat{D}$ a new Nash equilibrium $\hat{Q} \otimes \hat{Q}$ has emerged with pay-off $\$_{A}(\hat{Q}, \hat{Q})=\$_{B}(\hat{Q}, \hat{Q})=3$. Indeed, $\$_{A}(\hat{U}(\theta, \phi), \hat{Q})=\cos ^{2}(\theta / 2)\left(3 \sin ^{2} \phi+\cos ^{2} \phi\right) \leq 3$ for all $\theta \in[0, \pi]$ and $\phi \in[0, \pi / 2]$ and analogously $\$_{B}\left(\hat{Q}, \hat{U}_{B}\right) \leq$ $\$_{B}(\hat{Q}, \hat{Q})$ for all $\hat{U}_{B} \in S$ such that no player can gain from unilaterally deviating from $\hat{Q} \otimes \hat{Q}$. It can be shown that $\hat{Q} \otimes \hat{Q}$ is a unique equilibrium, that is, rational reasoning dictates both players to play $\hat{Q}$ as their optimal strategy.

It is interesting to see that $\hat{Q} \otimes \hat{Q}$ has the property to be Pareto optimal [2], that is, by deviating from this pair of strategies it is not possible to increase the pay-off of one player without lessening the pay-off of the other player. In the classical game only mutual cooperation is Pareto optimal, but it is not an equilibrium solution. One could say that by allowing for quantum strategies the players escape the dilemma [14].

The alert reader may object that - very much like any quantum mechanical system can be simulated on a classical computer - the quantum game proposed here can be played by purely classical means. For instance, Alice and Bob each may communicate their choice of angles to the judge using ordinary telephone lines. The judge computes the values $P_{\sigma \sigma^{\prime}}$, tosses a four-sided coin which is biased on these values, and returns the pay-off according to the outcome of the experiment. While such an implementation yields the proper pay-off in this scenario four real numbers have to be transmitted. This contrasts most dramatically with our quantum mechanical model which is more economical as far as communication resources are concerned. Moreover, any local hidden variable model of the physical scheme presented here predicts inequalities for $P_{\sigma \sigma^{\prime}}$,

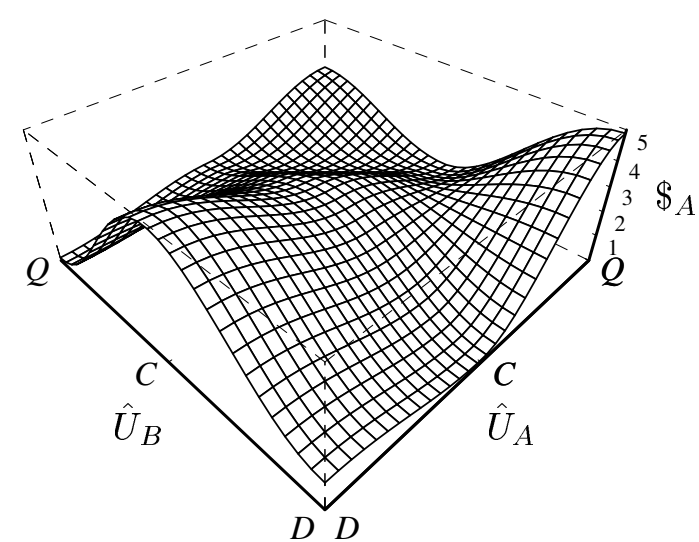

FIG. 3. Alice's pay-off for a maximally entangled game. The parametrization is chosen as in Fig. 2 .

as functions of the four angles $\theta_{A}, \theta_{B}, \phi_{A}$, and $\phi_{B}$, which are violated by the above expressions for the expected pay-off. We conclude that in an environment with limited resources, it is only quantum mechanics which allows for an implementation of the game presented here.

So far we have considered fair games where both players had access to a common strategic space. What happens when we introduce an unfair situation: Alice may use a quantum strategy, i.e., her strategic space is still $S$, while Bob is restricted to apply only "classical strategies" characterized by $\phi_{B}=0$ ? In this case Alice is well advised to play

$$
\hat{M}=\hat{U}(\pi / 2, \pi / 2), \quad \hat{M}=\frac{1}{\sqrt{2}}\left(\begin{array}{cc}
i & -1 \\
1 & -i
\end{array}\right),
$$

(the "miracle move"), giving her at least reward $r=3$ as payoff, since $\$_{A}(\hat{M}, \hat{U}(\theta, 0)) \geq 3$ for any $\theta \in[0, \pi]$, leaving Bob with $\$_{B}(\hat{M}, \hat{U}(\theta, 0)) \leq 1 / 2$ (see Fig. 4 (a)). Hence if in an unfair game Alice can be sure that Bob plays $\hat{U}(\theta, 0)$, she may choose "Always- $\hat{M}$ " as her preferred strategy in an iterated game. This certainly out-performs tit-for-tat, but one must keep in mind that the assumed asymmetry is essential for this argument.

It is moreover interesting to investigate how Alice's advantage in an unfair game depends on the degree of entanglement of the initial state vector $\left|\psi_{0}\right\rangle$. The minimal expected pay-off $m$ Alice can always attain by choosing an appropriate strategy $U_{A}$ is given by

$$
m=\max _{\hat{U}_{A} \in S} \min _{\hat{U}_{B} \in\{\hat{C}, \hat{D}\}} \$_{A}\left(\hat{U}_{A}, \hat{U}_{B}\right) .
$$

Alice will not settle for anything less than this quantity. Considering $m$ a function of the entanglement parameter $\gamma \in[0, \pi / 2]$ it is clear that $m(0)=1$ (since in this case the dominant strategy $\hat{D}$ is the optimal choice) while for maximal entanglement we find $m(\pi / 2)=3$ which is achieved by playing $\hat{M}$. Fig. 4 (b) shows $m$ as function of the entanglement parameter $\gamma$. We observe that $m$ is in fact a monotone increasing function of $\gamma$, and the maximal advantage is only accessible for maximal 
(a)

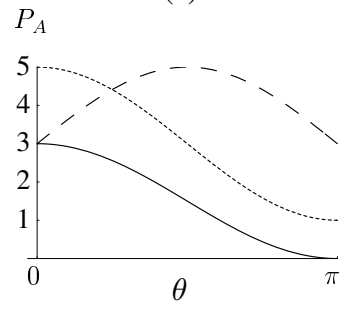

(b)

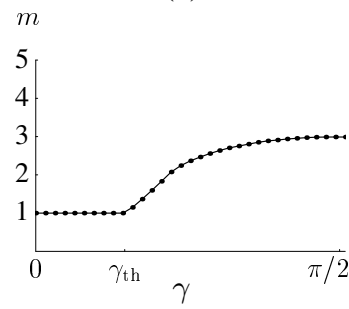

FIG. 4. Quantum versus classical strategies: (a) Alice's pay-off as a function of $\theta$ when Bob plays $\hat{U}(\theta, 0)(\hat{U}(0,0)=\hat{C}$ and $\hat{U}(\pi, 0)=$ $\hat{D})$ and Alice chooses $\hat{C}$ (solid line), $\hat{D}$ (dots) or $\hat{M}$ (dashes). (b) The expected pay-off Alice can always attain in an unfair game as a function of the entanglement parameter $\gamma$.

entanglement. Furthermore, Alice should deviate from the strategy $\hat{D}$ if and only if the degree of entanglement exceeds a certain threshold value $\gamma_{\text {th }}=\arcsin (1 / \sqrt{5}) \approx 0.464$. The observed threshold behavior is in fact reminiscent of a first order phase transition in Alice's optimal strategy: at the threshold she should discontinuously change her strategy from $\hat{D}$ to $\hat{Q}$.

Summarizing, we have demonstrated that novel features emerge if classical games like the Prisoners' Dilemma are extended into the quantum domain. We have introduced a correspondence principle which guarantees that the performance of a classical game and its quantum extension can be compared in an unbiased manner. Very much like in quantum cryptography and computation, we have found superior performance of the quantum strategies if entanglement is present.

This research was triggered by an inspiring talk of Artur Ekert on quantum computation. We also acknowledge fruitful discussions with S. M. Barnett, C. H. Bennett, R. Dum, T. Felbinger, P. L. Knight, H.-K. Lo, M. B. Plenio, A. Sanpera, and P. Zanardi. This work was supported by the DFG.
[1] J. von Neumann and O. Morgenstern, The Theory of Games and Economic Behaviour (Princeton University Press, Princeton, 1947).

[2] R. B. Myerson, Game Theory: An Analysis of Conflict (MIT Press, Cambridge, 1991).

[3] R. Axelrod, The Evolution of Cooperation (Basic Books, New York, 1984); R. Dawkins, The Selfish Gene (Oxford University Press, Oxford, 1976).

[4] C. H. Bennett, F. Bessette, G. Brassard, L. Salvail, and J. Smolin, J. Crypto. 5, 3 (1992)

[5] A. K. Ekert, Phys. Rev. Lett. 67, 661 (1991).

[6] N. Gisin and B. Huttner, Phys. Lett. A 228, 13 (1997).

[7] R. F. Werner, Phys. Rev. A 58, 1827 (1998).

[8] D. A. Meyer, Phys. Rev. Lett. 82, 1052 (1999).

[9] L. Goldenberg, L. Vaidman, and S. Wiesner, Phys. Rev. Lett. 82, 3356 (1999).

[10] Alice's reasoning goes as follows: "If Bob cooperates my payoff will be maximal if and only if I defect. If, on the other hand, Bob defects, my pay-off will again be maximal if and only if I defect. Hence I shall defect.".

[11] The Prisoners' Dilemma must be distinguished from its iterated versions where two players play the simple Prisoners' Dilemma several times while keeping track of the game's history. In a computer tournament conducted by Axelrod it was shown that a particular strategy tit-for-tat outperforms all other strategies [3].

[12] D. Deutsch, Proc. R. Soc. Lond. A 425, 73 (1989).

[13] Probabilistic strategies of this type are called mixed strategies in game theory.

[14] In a more general treatment one should include the possibility that each player can resort to any local operation quantum mechanics allows for. That is, each player may apply any completely positive mapping represented by operators $A_{i}$ (Alice) and $B_{i}$ (Bob), $i=1,2, \ldots$, respectively, fulfilling the tracepreserving properties $\sum_{i} A_{i}^{\dagger} A_{i}=1, \sum_{i} B_{i}^{\dagger} B_{i}=1$. It can then be shown that with these strategic options the unique Nash equilibrium of the main text is replaced by a continuous set and one isolated Nash equilibrium. This attracts the players' attention and will make the players expect and therefore fulfill it (the focal point effect [2]). The focal equilibrium is the one where Alice maps the initial state $\mathcal{J}\left|\psi_{0}\right\rangle\left\langle\psi_{0}\right| \mathcal{J}$ to $1 / 4$, and Bob chooses the same operation. This pair of strategies yields an expected pay-off of 2.25 to each player and is therefore again more efficient than the equilibrium in dominant strategies in the classical game. 